\title{
Le système éducatif colombien : structures, réformes et performances
}

Frédéric Mazières

\section{OpenEdition}

1 Journals

Édition électronique

URL : https://journals.openedition.org/ries/2053

DOI : 10.4000/ries.2053

ISSN : 2261-4265

Éditeur

France Education international

\section{Édition imprimée}

Date de publication : 1 septembre 2011

Pagination : $30-36$

ISBN : 978-2-85420-592-3

ISSN : $1254-4590$

Référence électronique

Frédéric Mazières, "Le système éducatif colombien : structures, réformes et performances ", Revue internationale d'éducation de Sèvres [En ligne], 57 | septembre 2011, mis en ligne le 01 septembre 2014, consulté le 05 juillet 2021. URL : http://journals.openedition.org/ries/2053 ; DOI : https://doi.org/ $10.4000 /$ ries. 2053

Ce document a été généré automatiquement le 5 juillet 2021.

(c) Tous droits réservés 


\title{
Le système éducatif colombien : structures, réformes et performances
}

\author{
Frédéric Mazières
}

1 La République de Colombie offre un double paradoxe politique. Contrairement à la plupart des pays sud-américains, elle n'a presque jamais connu de dictature. Cette stabilité générale ne doit pas faire oublier l'existence de nombreux conflits entre l'armée officielle et de nombreux groupes violents, les paramilitaires, les guérilleros, les narcotrafiquants. Malgré tout, l'État colombien se maintient. Il a même la réputation d'être un bon gestionnaire : la Colombie ne fait pas partie des pays très endettés, sa dette extérieure est en baisse ( $21,1 \%$ du PIB en $2009,18 \%$ en 2011), son taux de croissance est bon (4,7 \% du PIB en 2010), son marché est attractif, la Colombie étant le troisième pays plus compétitif d'Amérique latine. L'optique du gouvernement conservateur actuel est de poursuivre la politique sécuritaire amorcée par le gouvernement Uribe. Toutes les institutions de la société seront bénéficiaires, y compris le secteur éducatif.

\section{Structure décisionnelle et scolaire}

\section{Ministère de l'Éducation nationale et secrétariats d'Éducation}

2 Parmi toutes les fonctions que l'article 148 de la Loi générale d'éducation attribue au ministère de l'Éducation nationale (MEN), on peut sélectionner les trois fonctions suivantes :

- une fonction politique: conception, d'après des orientations gouvernementales et des valeurs éducatives qui peuvent différer selon les formations politiques au pouvoir, des politiques et des planifications éducatives. Ce sont toutes les politiques éducatives successivement mises en œuvre par les gouvernements qui forgent, progressivement, un système éducatif. D'autres valeurs peuvent intervenir dans sa genèse: philosophiques, 
religieuses, culturelles, historiques, économiques, démographiques. En Colombie, il existe, en outre, des paramètres incontournables: obédience libérale des politiques publiques, catholicisme influent, clientélisme ${ }^{1}$. Les hauts-fonctionnaires du MEN facilitent la mise en place des volontés gouvernementales en transformant les politiques énoncées en programmations ;

- une fonction normative : carrière des personnels (statuts, évolution), programmes ;

- une fonction évaluative: contrôle des institutions et des acteurs éducatifs. Le MEN colombien est dirigé par un ministre de l'Éducation nationale, ainsi que par deux viceministres : l'un chargé du primaire et du secondaire, l'autre du supérieur.

Le MEN peut déléguer certaines de ses compétences (ou fonctions) administratives aux secrétariats d'Éducation (départementaux, de district et municipaux) ou les partager. Alors que le ministère oriente le système éducatif dans sa globalité, les secrétariats sont responsables des prestations régionales ou municipales du service éducatif de la Nation.

\section{Structure scolaire}

L'organigramme du système éducatif colombien est composé de cinq niveaux d'éducation: éducation pré-scolaire; éducation de base; éducation médiane académique ou technique; éducation supérieure (pregrado): technique, technologique et professionnel ; éducation supérieure (posgrado) : spécialisation, maitrise et doctorat. Le taux d'espérance de vie scolaire est particulièrement faible. Il est passé, du primaire au supérieur, de 11,6 (1999) à 13,3 (2008)². Cependant, entre 1980 et 2000, le nombre d'années de scolarité obligatoire a plus que doublé, passant de cinq à onze années.

\section{Dispositifs légaux et administratifs}

\section{Constitution politique de 1991}

Cette Constitution régit l'organisation juridico-politique de la Colombie. L'éducation, qui y est définie comme un des axes majeurs de l'action de l'État, est :

- un droit fondamental de l'individu ;

- un service public ;

- un moyen de réaliser la justice sociale.

6 La Constitution vise un idéal : l'État social de droit. À cet effet, un système complexe d'actions de surveillance et d'évaluation a été mis en place, notamment dans le secteur supérieur (article 5) : «il incombe à l'État d'exercer une très grande surveillance de l'éducation afin de veiller à sa qualité, au respect de ses finalités et dans le but d'assurer aux personnes éduquées la meilleure formation morale, intellectuelle et physique ». La Constitution a favorisé la décentralisation du système éducatif en favorisant sa municipalisation (1980-1991), puis sa départementalisation (1991-2001).

\section{Loi générale d’Éducation du 8 février 1994 (ou Loi 115)}

7 La Loi 115 a permis les aménagements suivants : 


\section{Bilan régulier des planifications gouvernementales}

8 Tous les dix ans, il est prévu la rédaction d'un Plan national de développement éducatif (PND) pour faire le point sur les avancées du système éducatif. À l'occasion de celui de 1996-2005 (Balance del Plan Decenal de Educación), de nombreux acteurs de la société colombienne ${ }^{3}$ se sont mobilisés pour tenter d'élaborer une politique éducative plurielle. Ce cas de démocratie participative, qui aboutit à la rédaction d'une synthèse des propositions, permit aux fonctionnaires de l'administration centrale de comprendre les intentions et les stratégies des acteurs éducatifs, et notamment des syndicats, qui pouvaient s'opposer à des réformes jugées trop libérales. Ainsi, la Fédération colombienne des éducateurs (Fecode) parvint à faire échouer, au début des années 2000, le PND Cambio para construir la Paz de l'administration Pastrana. La réussite du projet actuel de réforme de l'éducation supérieure passera par un accord avec la Fecode.

\section{Réformes structurelles}

La décentralisation ${ }^{4}$ du système éducatif colombien est équilibrée : la répartition entre les pouvoirs centraux, régionaux et locaux reste homogène. Le MEN a gardé ses prérogatives sur l'essentiel :

- formulation des politiques éducatives ;

- définition des paramètres du service éducatif (ressources humaines);

- définition des règles et des mécanismes pour l'évaluation, la formation et les concours des professions enseignantes;

- calcul des budgets ;

- contrôle des coûts, des inscriptions, des pensions et des droits académiques ;

- évaluation de la qualité de l'éducation ;

- définition des programmes et des politiques éducatives ;

- inspection et vigilance.

\section{Vers une privatisation de l'éducation colombienne?}

10 La Colombie, encouragée par des organismes multilatéraux (Banque mondiale, Fonds monétaire international), est devenue, au fil des gouvernements conservateurs, une terre favorable au libéralisme en éducation. Les réformes, les lois et les plans éducatifs colombiens mènent vers la privatisation, voire vers la capitalisation du système éducatif colombien, avec, par exemple, des partenariats public-privé et/ou des financements d'actions publiques par des capitaux privés.

Le modèle éducatif libéral repose sur les concepts suivants : commercialisation des services éducatifs (OMC), rapport coût-bénéfice/efficacité, financement de l'éducation, accès et couverture, équité, qualité, efficacité, évaluation. Les aspects pédagogiques deviennent secondaires. Le quantitatif (économie et statistiques ${ }^{5}$ ) l'emporte sur le qualitatif (pédagogique et culturel). Les États endettés sont invités, par les organismes internationaux, et dans le cadre de réajustements structurels, à se décharger de responsabilités éducatives devenues trop onéreuses : environ $16 \%$ du budget d'un État ${ }^{6}$, 7 . 
l'tat colombien est endetté. Les guerres contre ses ennemis coûtent cher. Les déficits se creusent entre les besoins éducatifs de la Nation et les crédits accordés par les ministères des finances. À partir de ces nouvelles tendances, les derniers gouvernements colombiens, libéraux ${ }^{8}$ ont donc procédé aux aménagements suivants :

- la Loi 715 de 2001 (chapitre III, articles 6 et 7) prévoit que les entités territoriales administrent les fonds provenant du SGP (Sistema General de Participaciones), ceux, notamment, destinés au secteur éducatif. Les subventions passent de l'État aux départements, puis des départements aux municipalités. En 2007, Le secteur éducatif représentait 58,05\% du SGP9. Les budgets affectés aux municipalités sont conséquents. La décentralisation peut favoriser la corruption locale. L'argent manquant, les parents d'élèves assument les coûts et interviennent de plus en plus dans la pédagogie et dans la gestion des écoles ;

- la Loi 60 de 1993 prévoyait que l'on ventilât les fonds prévus pour le secteur éducatif en fonction des rentrées d'argent de la Nation. Depuis les réformes budgétaires des années 2000 et 2001, les budgets destinés au secteur éducatif varient en fonction des intérêts politiques du gouvernement au pouvoir et des évolutions macro-économiques du marché mondial. Les subventions sont calculées en fonction du nombre d'élèves scolarisés et effectivement accueillis. Les secteurs du privé et du public se livrent à d'impitoyables compétitions pour récupérer les élèves. Cependant, les dépenses publiques pour l'éducation (primaire et secondaire) ont progressé : de 3,2\% en 2000 à 3,90\% en 2010 (site du MEN colombien, statistiques).

Dans le secteur supérieur, la participation de l'État reste insuffisante : $1 \%$ en 2000 et $1,1 \%$ en 2010 . Une complète privatisation du secteur supérieur se profile : le nombre d'établissements privés du supérieur, entre 1990 et 2002, avait bien plus augmenté que celui des établissements publics ${ }^{10}$, alors que ces derniers (et leur qualité) sont protégés par l'article 98 de la Loi 30 de 1992. Mais, comme les gens veulent une bonne éducation, «cela alimente le système des universités-garage qui trichent, à l'abri [de la loi] de l'autonomie [des universités] et de la corruption des autorités ${ }^{11}$. Déguisées en fondations ou en sociétés, ces organismes se sont accrus trois fois plus vite que les vraies universités ces vingt dernières années ». Le projet de réforme de l'éducation supérieure propose une participation de l'État de $3 \%$, de 2014 à 2019. Il est soutenu par les établissements d'enseignement supérieur.

\section{Privatisation et décentralisation}

14 Décentraliser ne conduit pas automatiquement à une amélioration d'un système éducatif mais aussi à des schémas de privatisation et de cofinancement (public/privé) du secteur éducatif : gestion de collèges par des organismes privés ; création de collèges au statut hybride (ou fusionnés). En Colombie, entre 2000 et 2002, on recensait ainsi les fusions de 771 institutions éducatives ${ }^{12}$. Ces différents arrangements avec le secteur privé devaient permettre, selon les experts de l'administration libérale, d'améliorer le taux de couverture scolaire de $90 \%$ (sur la base d'un enseignant pour 30 élèves). Et en effet, entre 2002 et 2008, les taux de couverture ont augmenté de $57 \%$ à $70 \%$, notamment dans le secondaire (MEN colombien), mais au prix de fermetures d'établissements scolaires et de licenciements massifs de professeurs, cette dernière mesure étant facilitée par la mise en place d'un nouveau statut moins avantageux que le précédent. 


\section{Modification du statut des enseignants}

Avec la Loi 715 de 2001 (chapitres 2 à 4) et le Décret-Loi 1278 de 2002 (chapitre 4), l'administration Pastrana a créé un nouveau statut (Estatuto de Profesionalización Docente) qui introduit, dans le monde enseignant, les notions de mérite et de flexibilité du travail et entraîne les aménagements suivants :

-évaluations périodiques et permanentes des enseignants ;

- calcul des salaires, en fonction des résultats obtenus ;

- réduction du nombre de promotions ;

- augmentation du ratio élèves/enseignants. Cette mesure permet une augmentation de la couverture scolaire. Mais des enseignants sont congédiés et les élèves fuient des classes surchargées. La mauvaise répartition des professeurs explique l'abandon scolaire. Il existe également des causes plus graves et plus profondes : la pauvreté et la guerre civile.

Actuellement, des négociations tripartites entre le Congrès, le MEN et la Fecode sont en cours pour évaluer la possibilité de créer un statut unique des enseignants (Estatuto Unico Docente). Le projet de la ministre, Madame Campo, est de proposer à la fois une évolution et une synthèse des anciens statuts.

\section{Performances du système éducatif colombien}

17 Au regard des Objectifs du Millénaire pour le Développement (OMD), les performances colombiennes sont encore insuffisantes : le taux d'analphabétisme était de 3,77\% en 1992 et de $2,1 \%$ en 2007 ; l'OMD est de $1 \%$ d'ici 2015 . Cependant, des progrès ont été réalisés : entre 2002 et 2008, ce taux est passé de $2,56 \%$ à $2,01 \%$ (MEN colombien). Le taux de redoublement était de $6,1 \%$ en 1992 et de $4 \%$ en 2007 ; l'OMD est de $2,3 \%$.

Entre 1980 et 1996, la participation de l'État aux dépenses publiques pour l'ensemble du secteur éducatif avait progressé de $2,4 \%$ du PNB à $4,5 \%{ }^{13}$. En 2010, elle est en baisse à $3,9 \%$ du PIB (Unesco, 2011), contre 4,8\% en 2005 (Unesco, 2007), mais comparable à celles de pays voisins à l'économie compétitive de la zone sud-américaine ${ }^{14}$. La privatisation du système reflète-t-elle l'abandon des pouvoirs publics? Les crédits éducatifs provenant des organisations internationales augmentent: entre 2002 et 2006, les aides de l'Unesco et de l'Unicef ont progressé, et celles de l'Unesco ont plus que doublé, passant de 337000 à 700000 dollars USD ${ }^{15}$. La tradition colombienne est de ventiler les budgets éducatifs plutôt vers le supérieur ( $26 \%$ ), au détriment du primaire $(12,4 \%)$ et du secondaire $(14,8 \%)^{16}$. Le taux net de scolarité du secondaire est assez faible (71\%), contre $82 \%$ au Brésil et $85 \%$ au Chili (Unesco, 2010). Le système éducatif colombien peine à résoudre le phénomène de l'abandon scolaire, notamment dans les campagnes, où le MEN enregistrait en 2008 un taux de fréquentation de $24,38 \%$ (éducation de base et médiane). En 2002, ce taux était de 23,45\%. Les progrès sont lents. Alors que le taux d'abandon scolaire est, en 2006 , de $4 \%$ en ville, il est de $5 \%$ dans les campagnes (éducation de base et médiane, Corpoeducación, 2006). Les différentes formes de violence contre les élèves et les professeurs, dans les nombreuses zones de non droit que compte la Colombie, expliquent ces résultats, ainsi que le nombre important d'analphabètes en Colombie : en 2005, ils étaient environ 2250000 (Unesco, 2007), et le taux d'analphabétisme était, en 2007, de 6,6 \% (MEN colombien) ${ }^{17}$. 
19 Alors qu'au Brésil et au Chili le ratio élèves/enseignants dans le secondaire est de 17 et 24, il s'élève à 26 en Colombie (Unesco, 2011).

En matière de qualité et de réussite aux tests nationaux et internationaux, les résultats au PISA 2006, que ce soit en sciences, en lecture ou mathématiques, étaient mauvais, notamment par rapport à ceux des autres élèves sud-américains. Le système éducatif colombien ne semblait pas bien assurer la transmission des savoirs, constat confirmé par les taux bruts de diplômés du secteur supérieur ${ }^{18}$. Cependant, même si les résultats en lecture restent préoccupants (413 points), les chiffres au PISA 2009 sont meilleurs. La Colombie enregistre même l'une des meilleures progressions mondiales. Si l'on se base sur le taux de transition entre l'école primaire et le secondaire, les résultats des élèves colombiens sont plus qu'estimables : 98 \% (Unesco, 2011). Entre 2004 et 2005, il était même de 100 \% (Unesco, 2007) ${ }^{19}$.

21 En ce qui concerne la mise en place d'une éducation équitable, les contextes sociaux sud-américains ne sont pas favorables. Le pourcentage de personnes pauvres augmente, notamment dans les villes. L'Amérique latine est la région qui affiche les taux de distribution de la richesse les plus inégalitaires du monde. Si on veut étudier en Colombie, il était encore préférable, en 2006, d'habiter dans une ville et d'être riche. Seuls $11 \%$ des enfants de la première catégorie de revenus (catégorie la plus pauvre) sont étudiants entre 18 et 24 ans contre $52 \%$ des étudiants de la $10^{\mathrm{e}}$ catégorie (la plus riche, Corpoeducación, 2006).

Pour relancer le goût des apprenants et des enseignants pour l'école primaire et secondaire, des pédagogues colombiens, dans les années 1970 et 1980, avaient imaginé une méthode d'auto-apprentissage et d'auto-enseignement, destinée aux élèves du primaire: la méthode Escuela Nueva. Une majorité d'écoles rurales l'ont, depuis, adoptée. Des pays voisins, comme le Honduras et le Venezuela, ont même envoyé des enseignants se former à cette nouvelle approche. Cette méthode originale a non seulement permis l'amélioration de la qualité de l'enseignement mais aussi l'amélioration des performances des indicateurs de résultats du système colombien (taux de transition).

Les responsables du système éducatif colombien sont méritants. La situation politique et sécuritaire est, dans certaines régions du pays, chaotique. Et pourtant, des écoles bilingues et biculturelles, amérindiennes et afro-colombiennes, naissent régulièrement, y compris dans les régions les plus reculées du pays. De nombreux défis restent encore à relever : améliorer l'accès à l'éducation aux populations rurales et/ou déplacées par les conflits armés; améliorer le statut des enseignants; consolider les processus de décentralisation et d'autonomie, en responsabilisant, encore davantage, les autorités éducatives et administratives locales ; augmenter la participation financière de l'État au secteur public. 


\section{BIBLIOGRAPHIE}

Balance del Plan Decenal de Educación 1996-2005. La educación un compromiso de todos, MEN, Bogotá, 2006.

Hay avances pero quedan desafíos, informe de progreso educativo Colombia, Corpoeducación-fondación Corona-Preal, Bogotá, 2006.

Site du MEN colombien (statistiques) : http://201.234.245.149/seguimiento/estadisticas/ (consulté en juillet 2011).

Recueil de données mondiales sur l'éducation 2010, Institut statistique de l'Unesco, Unesco, Paris.

PISA (Colombie) : http://www.icfes.gov.co/pisa/phocadownload/pisa2009/infome_pisa_2009.pdf (consulté le 07 juin 2011).

\section{NOTES}

1. Constat d'un universitaire: "à un État clientéliste correspond aussi une éducation corporatiste; la preuve de ceci réside dans l'histoire de l'éducation en Colombie qui, depuis ses origines, fut soumise aux intérêts des partis politiques, à l'institution ecclésiastique et aux associations privées". [Traduit par F. Mazières]. Libreros Daniel. Tensiones de las políticas educativas en Colombia, balances y perspectivas. Universidad Pedagógica Nacional, Bogotá, 2002, p. 87. Cette configuration clientéliste peut bloquer les réformes et/ou les projets qui iraient contre les intérêts des corporations les plus puissantes (consortiums éducatifs privés).

2. En 2008, celui du Brésil est de 14, celui du Chili est de 14,5 (Unesco 2011).

3. Gouvernement, secteur productif, médias, communauté éducative.

4. Avant la Constitution de 1991, il s'agit d'une déconcentration (décentralisation mineure); après la Constitution, c'est une décentralisation.

5. Taux de réussite aux examens, taux de redoublement.

6. Carnoy Martin, 1999. Mondialisation et réforme de l'éducation; ce que les planificateurs doivent savoir, Unesco, Paris, p. 46.

7. Ainsi, la Banque mondiale a prêté assistance « au Salvador : pour inciter les banques privées à contribuer au financement des bourses d'études ; [à l'] Indonésie : pour stimuler la concurrence entre établissements publics et privés [...] », Belfield Clive R., Levin Henry M. : La privatisation de l'éducation : causes, effets et conséquences pour la planification, IIPE-Unesco, Paris, 2003, p. 37.

8. Les réformes libérales de l'administration Pastrana (1998-2002) se sont poursuivies sous l'administration Uribe (2002-2010). L'actuel Président colombien, Juan Manuel Santos, est un ancien ministre d'Uribe.

9. Loi 1176 de 2007 , article 4 .

10. Cardenas Jorge Hernán. Estudios sobre la Educación Superior en Colombia, la educación superior privada en Colombia, Ascun-Unesco, Bogotá, 2005.

11. Notamment dans le secteur supérieur et dans les zones rurales isolées.

12. Plan stratégique d'éducation 2000-2002, MEN.

13. Unesco. Bilan des 20 ans du Projet Majeur dans le domaine de l'éducation en Amérique latine et dans les Caraïbes, Paris, 2001.

14. Brésil : $5,2 \%$; Chili : $3,4 \%$ (Unesco 2011).

15. Site de l'Agence présidentielle pour l'action sociale et la coopération internationale. 
16. Nature des dépenses, Unesco, 2011 (résultats de 2008). On retrouvait les mêmes proportions en 2005 : 19,5 (primaire) ; 18,4 (secondaire) ; 24,6 (supérieur) : Unesco, 2007.

17. Le taux du Chili est de $1,4 \%$ (éducation de base et médiane). Celui du Brésil est de $10 \%$.

18. Entre $12 \%$ et $15 \%$. Les taux du Brésil, par exemple, oscillent entre $16 \%$ et $27 \%$ : Unesco, 2011.

19. Le Brésil et le Chili avaient des taux inférieurs : 81 et 97.

\section{INDEX}

Index géographique : Colombie

Keywords : educational reform, school system

Palabras claves : reforma de la educación, sistema escolar

Mots-clés : réforme de l'enseignement, système scolaire

\section{AUTEUR}

\section{FRÉDÉRIC MAZIÈRES}

Docteur en didactique des langues et des cultures de l'Université Paris III-Sorbonne Nouvelle. Il a enseigné les lettres modernes de 1998 à 2010 dans le réseau culturel français à l'étranger (Colombie, Équateur), ainsi que dans les Caraïbes. Il intervient à l'Université de Provence (master 2) sur les politiques culturelles du ministère français des Affaires étrangères et européennes, et plus précisément sur la politique linguistique extérieure de la France. 